Article

\title{
Lag Synchronization of Complex Lorenz System with Applications to Communication
}

\section{Fangfang Zhang}

School of Electrical Engineering and Automation, Qilu University of Technology, Jinan 250353, China; E-Mail: zff@qlu.edu.cn

Academic Editor: Raúl Alcaraz Martínez

Received: 25 March 2015 / Accepted: 2 July 2015 / Published: 15 July 2015

\begin{abstract}
In communication, the signal at the receiver end at time $t$ is the signal from the transmitter side at time $t-\tau(\tau \geq 0$ and it is the lag time) as the time lag of transmission. Therefore, lag synchronization (LS) is more accurate than complete synchronization to design communication scheme. Taking complex Lorenz system as an example, we design the LS controller according to error feedback. Using chaotic masking, we propose a communication scheme based on LS and independent component analysis (ICA). It is suitable to transmit multiple messages with all kinds of amplitudes and it has the ability of anti-noise. Numerical simulations verify the feasibility and effectiveness of the presented schemes.
\end{abstract}

Keywords: lag synchronization; complex chaotic system; communication; error feedback

\section{Introduction}

During actual applications, there always exists time lag. Taking the telephone communication system as an example, the sound one person hears on the receiver end at time $t$ is the sound produced on the transmitter end at time $t-\tau$ ( $\tau \geq 0$ and it is the lag time). There also exists time delay when the information signal transmits from the sender side to the receiver side in secure communication. "Many experimental investigations and computer simulations of chaos synchronization in unidirectional coupled external cavity semiconductor lasers [1,2] have demonstrated the presence of lag time between the drive and response lasers intensities" [3]. "The similar experiments for chaotic circuits have also demonstrated the complete synchronization (CS), i.e., the states of two chaotic systems remain identical in the course of temporal evolution, is practically impossible for the presence of the signal transmission time and evolution time of response system itself." [3] 
Therefore, the requirement that the response system synchronizes the drive system at exactly the same time is not suitable. Lag synchronization (LS) means the state of the response system at time $t$ is asymptotically synchronous with the drive system at time $t-\tau$, i.e., $\lim _{t \rightarrow \infty}\|\mathbf{x}(t)-\mathbf{y}(t-\tau)\|=0$ where $\mathbf{x}(t)$ and $\mathbf{y}(t)$ are the states of the response and drive systems, respectively. Thus, LS is more rigorous than CS in practice and CS is just a special case of LS with $\tau=0$ [4].

As Fowler et al. [5] proposed the complex Lorenz equations, complex systems have played a vital role in many subjects of physics [6], most notably chaos-communication, where "the complex variables (doubling the number of variables) increase the contents and security of the transmitted information" [7], i.e., "complex nonlinear system can increase the capacity of transmitted contents or thought the expense of the transmission capacity in exchange for the greater security" [8]. Hence, the synchronization of complex chaotic systems [9-19] has aroused great interest in the last few decades. Emad E. Mahmoud et al. had investigated LS of complex nonlinear chaotic systems using passive control [3] and active control [14], respectively. However, the methods they used are complicated, and they did not apply it to secure communication.

Now there are a great many papers [20-30] about communication schemes based on real chaotic system, while the communication schemes based on complex chaotic systems has seldom been researched except Refs. [8,31-34]. Ref. [8] designed "an improved switch-modulated digital secure communication system based on a hybrid synchronization scheme in coupled fractional-order complex Chen system". Ref. [31] proposed "an improved digital secure communication scheme using hybrid modulus-phase synchronization of hyperchaotic complex systems". Ref. [32] adopted projective synchronization to design communication scheme based on chaotic modulation, while Ref. [33] employed complex function projective synchronization and Ref. [34] employed self-time-delay synchronization of time-delay coupled complex Lorenz chaotic system to design communication scheme based on chaotic masking. However, to our best knowledge, there is no paper on the chaos-communication schemes based on LS of complex chaotic systems. In fact, as the time delay of transmission, it is much closer to the real situation and more accurate to describe the synchronization between the sender end and the receiver end based on LS. It is one natural advantage in theory.

The fundamental requirement of chaotic masking is that the power spectrum of message "should be buried into that of the chaotic signal" [23]. Consequently, "the dynamic range of the messages has to be significantly smaller than that of the chaotic scalar" [23]. Furthermore, "the addition of a message signal to the chaotic scalar at the transmitter can degrade the quality of the synchronization between the transmitter and the receiver, and even result in the loss of synchronization if the amplitude of the message is too large" [23]. In fact, the amplitude of the message is larger than that of chaotic signal in many practical situations. Hence, it is necessary to study the transmission of multiple messages with larger amplitudes. In this paper, we solve this problem in a way.

Recently, complex variables is widely applied to a number of nonlinear systems, for example detuned laser systems [6], coupled map lattices [35] and Julia sets [36]. Complex Lorenz system is one of the most common complex chaotic systems, and has been used to describe rotating fluids $[6,37,38]$, disk dynamos [39], etc. Moreover, it is believed that the complex Chen and Lü systems belong to the same class of the Lorenz equation [7,40-43] and they have similar properties. Therefore, taking complex Lorenz chaotic system as example, we design LS controller by error feedback (different from 
Refs. [3,14] and it is simpler than them), and then investigate the communication schemes based on LS to transmit multiple messages considering the influence of noises. The rest of this paper is organized as follows: we design the LS controller in Section 2. The communication scheme based on LS is discussed in Section 3, and numerical simulations are given in Section 4. In the end, we summarize some conclusions in Section 5.

\section{The LS Controller of Complex Lorenz Systems}

We are to realize the lag synchronization of complex Lorenz systems employing error feedback in this section. First, we give the following Lemma 1 and Theorem 1 which will play an important role in proving Theorem 2.

Lemma 1. [44] If $n \times n$ real matrix is the row (column) diagonally dominant matrix where all of the diagonal elements are negative, then all eigenvalues of this matrix have negative real parts.

Theorem 1. For given linear time-varying system

$$
\dot{\mathbf{z}}(t)=\mathbf{A}(t) \mathbf{z}(t)
$$

where $\mathbf{z}(t) \in R^{n}, \mathbf{A}(t)$ is a $n \times n$ time-varying matrix. Its elements are bounded for all $t \geq 0\left(a_{\min }<\right.$ $\left|a_{i j}(t)\right|<a_{\max }$ where $a_{\min }>0$ and $a_{\max }>0$ and they are independent of $\left.t\right)$. If there exists positive definite matrix $\mathbf{P}$ to ensure $\mathbf{A}^{\mathrm{T}}(t) \mathbf{P}+\mathbf{P A}(t)$ negative definite, then $\mathbf{z}(t)=0$ is the global equilibrium point with exponential asymptotic stability of system (1).

Proof. For positive definite matrix $\mathbf{P}$, we adopt Lyapunov function as

$$
V(\mathbf{z}(t))=\mathbf{z}^{\mathrm{T}}(t) \mathbf{P} \mathbf{z}(t)
$$

We employ $\mathbf{z}, \dot{\mathbf{z}}$ instead of $\mathbf{z}(t), \dot{\mathbf{z}}(t)$, respectively, for simplicity. The similar thereinafter.

Its derivative along $\dot{z}$ is

$$
\dot{V}(\mathbf{z})=\dot{\mathbf{z}}^{\mathrm{T}} \mathbf{P} \mathbf{z}+\mathbf{z}^{\mathrm{T}} \mathbf{P} \dot{\mathbf{z}}=\mathbf{z}^{\mathrm{T}}\left[\mathbf{A}^{\mathrm{T}}(t) \mathbf{P}+\mathbf{P A}(\mathbf{t})\right] \mathbf{z} .
$$

If there exists positive definite matrix $\mathbf{P}$ to ensure $\mathbf{A}^{\mathrm{T}}(t) \mathbf{P}+\mathbf{P A}(t)$ negative definite and $\mathbf{A}(t)$ is bounded for all $t>0$, we set $-k(k>0)$ as the maximum eigenvalue of the matrix $\mathbf{A}^{\mathrm{T}}(t) \mathbf{P}+\mathbf{P A}(t)$, then

$$
\dot{V}(\mathbf{z}) \leq-k\|\mathbf{z}\|^{2}
$$

As $\mathbf{P}$ is independent of time, $\mathbf{z}=0$ is the globally equilibrium point with exponential asymptotic stability of system (1). The proof is completed.

Theorem 2. Consider the following drive complex Lorenz chaotic system (5) and the response complex Lorenz chaotic system (6)

$$
\left\{\begin{array}{l}
\dot{y}_{1}=a_{1}\left(y_{2}-y_{1}\right) \\
\dot{y}_{2}=a_{2} y_{1}-y_{1} y_{3}-y_{2} \\
\dot{y}_{3}=-a_{3} y_{3}+(1 / 2)\left(\bar{y}_{1} y_{2}+y_{1} \bar{y}_{2}\right)
\end{array}\right.
$$


where $y_{1}=u_{1}+j u_{2}$ and $y_{2}=u_{3}+j u_{4}$ are complex state variables, $y_{3}=u_{5}$ is real state variable. $\left(a_{1}, a_{2}, a_{3}\right)^{\mathrm{T}}$ is the real parameter vector. The overbar $\bar{y}_{1}\left(\bar{y}_{2}\right)$ represents the complex conjugate of $y_{1}\left(y_{2}\right)$;

$$
\left\{\begin{array}{l}
\dot{x}_{1}=a_{1}\left(x_{2}-x_{1}\right)+v_{1} \\
\dot{x}_{2}=a_{2} x_{1}-x_{1} x_{3}-x_{2}+v_{2}, \\
\dot{x}_{3}=-a_{3} x_{3}+(1 / 2)\left(\bar{x}_{1} x_{2}+x_{1} \bar{x}_{2}\right)+v_{3}
\end{array}\right.
$$

where $x_{1}=u_{1}^{\prime}+j u_{2}^{\prime}$ and $x_{2}=u_{3}^{\prime}+j u_{4}^{\prime}$ are complex state variables, $x_{3}=u_{5}^{\prime}$ is real state variable, $\mathbf{v}=\left(v_{1}, v_{2}, v_{3}\right)^{\mathrm{T}}$ is the control vector.

Set the errors $e_{1}=y_{1}(t-\tau)-x_{1}(t), e_{2}=y_{2}(t-\tau)-x_{2}(t)$ and $e_{3}=y_{3}(t-\tau)-x_{3}(t)$ where $\tau$ is the constant time lag. If we adopt the controller

$$
\left\{\begin{array}{l}
v_{1}=k_{1} e_{1}, \\
v_{2}=k_{2} e_{2}, \\
v_{3}=k_{3} e_{3},
\end{array}\right.
$$

then there exist real constants $k_{1}, k_{2}, k_{3} \in R$ satisfying

$$
\lim _{t \rightarrow \infty}\|\mathbf{e}\|^{2}=0
$$

i.e., we realize the LS between system (5) and system (6).

Proof. As $y_{1}(t-\tau)=u_{1}(t-\tau)+j u_{2}(t-\tau)$ and $x_{1}(t)=u_{1}^{\prime}(t)+j u_{2}^{\prime}(t)$, then $e_{1}=y_{1}(t-\tau)-$ $x_{1}(t)=u_{1}(t-\tau)-u_{1}^{\prime}(t)+j\left[u_{2}(t-\tau)-u_{2}^{\prime}(t)\right]$. For simplicity, we adopt $y_{1 \tau}, u_{1 \tau}, u_{2 \tau}$ instead of $y_{1}(t-\tau), u_{1}(t-\tau), u_{2}(t-\tau)$ respectively, and the similar thereinafter.

Set $e_{u 1}=u_{1 \tau}-u_{1}^{\prime}, e_{u 2}=u_{2 \tau}-u_{2}^{\prime}, e_{u 3}=u_{3 \tau}-u_{3}^{\prime}, e_{u 4}=u_{4 \tau}-u_{4}^{\prime}, e_{u 5}=u_{5 \tau}-u_{5}^{\prime}$, then $e_{1}=e_{u 1}+j e_{u 2}, e_{2}=e_{u 3}+j e_{u 4}$ and $e_{3}=e_{u 5}$. From (5)-(7), we obtain the following error system in real version

$$
\left\{\begin{array}{l}
\dot{e}_{u 1}=a_{1}\left(e_{u 3}-e_{u 1}\right)-k_{1} e_{u 1} \\
\dot{e}_{u 2}=a_{1}\left(e_{u 4}-e_{u 2}\right)-k_{1} e_{u 2} \\
\dot{e}_{u 3}=a_{2} e_{u 1}-\left(k_{2}+1\right) e_{u 3}-u_{5}^{\prime} e_{u 1}-u_{1 \tau} e_{u 5} \\
\dot{e}_{u 4}=a_{2} e_{u 2}-\left(k_{2}+1\right) e_{u 4}-u_{5}^{\prime} e_{u 2}-u_{2 \tau} e_{u 5} \\
\dot{e}_{u 5}=-a_{3} e_{u 5}-k_{3} e_{u 5}+\left(u_{1}^{\prime} e_{u 3}+u_{3 \tau} e_{u 1}+u_{2}^{\prime} e_{u 4}+u_{4 \tau} e_{u 2}\right) .
\end{array}\right.
$$

We can rewrite (9) as

$$
\dot{\mathbf{e}}_{u}=\mathbf{A}(t) \mathbf{e}_{u}
$$

where

$$
A(t)=\left(\begin{array}{ccccc}
-a_{1}-k_{1} & 0 & a_{1} & 0 & 0 \\
0 & -a_{1}-k_{1} & 0 & a_{1} & 0 \\
a_{2}-u_{5}^{\prime} & 0 & -k_{2}-1 & 0 & u_{1 \tau} \\
0 & a_{2}-u_{5}^{\prime} & 0 & -k_{2}-1 & u_{2 \tau} \\
u_{3 \tau} & u_{4 \tau} & u_{1}^{\prime} & u_{2}^{\prime} & -a_{3}-k_{3}
\end{array}\right)
$$


If we select $P=I$, then

$$
\mathbf{A}^{\mathrm{T}}(t)+\mathbf{A}(t)=\left(\begin{array}{ccccc}
-2\left(a_{1}+k_{1}\right) & 0 & a_{1}+a_{2}-u_{5}^{\prime} & 0 & u_{3 \tau} \\
0 & -2\left(a_{1}+k_{1}\right) & 0 & a_{1}+a_{2}-u_{5}^{\prime} & u_{4 \tau} \\
a_{1}+a_{2}-u_{5}^{\prime} & 0 & -2\left(k_{2}+1\right) & 0 & u_{1 \tau}+u_{1}^{\prime} \\
0 & a_{1}+a_{2}-u_{5}^{\prime} & 0 & -2\left(k_{2}+1\right) & u_{2 \tau}+u_{2}^{\prime} \\
u_{3 \tau} & u_{4 \tau} & u_{1 \tau}+u_{1}^{\prime} & u_{2 \tau}+u_{2}^{\prime} & -2\left(a_{3}+k_{3}\right)
\end{array}\right) .
$$

As $u_{i}, u_{i}^{\prime}(i=1,2, \cdots, 5)$ are bounded for chaotic system, $A(t)$ is bounded, and $a_{\min }<\left|a_{i j}(t)\right|<a_{\max }$ where $a_{\min }>0$ and $a_{\max }>0$. According to Lemma 1 , there exist $k_{1}>a_{\max }-a_{1} / 2, k_{2}>\left(3 a_{\max }+\right.$ $\left.a_{1}\right) / 2-1$ and $k_{3}>3 a_{\max }-a_{3}$ to ensure that $\mathbf{A}^{\mathrm{T}}(t)+\mathbf{A}(t)$ is a row diagonally dominant matrix where all of the diagonal elements are negative, then all eigenvalues of real symmetric matrix $\mathbf{A}^{\mathrm{T}}(t)+\mathbf{A}(t)$ are negative. Therefore, according to Theorem 1, we obtain $\mathbf{e}_{u}=0$ is the global equilibrium point with exponential asymptotic stability of system (10). It means (8) holds true, i.e., we realize the LS between complex Lorenz systems (5) and (6). The proof is completed.

Remark 1. The conditions of $k_{1}, k_{2}, k_{3}$ are sufficient, but not necessary.

Remark 2. The convergence rate of error system (10) is flexibly adjusted by selecting different $k_{1}, k_{2}, k_{3}$. Moreover, the value of $k_{1}, k_{2}, k_{3}$ is related to the ability of anti-noise and robustness in a way.

\section{A Communication Scheme Based on LS}

We design communication scheme based on LS in theory this section. We employ $L 1$ as the transmitter and $L 2$ as the receiver by means of the following complex chaotic systems

$$
L 1:\left\{\begin{array}{l}
\dot{y}_{1}=a_{1}\left(y_{2}-y_{1}\right)+b h+D \varepsilon(t)+j D \varepsilon(t), \\
\dot{y}_{2}=a_{2} y_{1}-y_{1} y_{3}-y_{2} \\
\dot{y}_{3}=-a_{3} y_{3}+(1 / 2)\left(\bar{y}_{1} y_{2}+y_{1} \bar{y}_{2}\right), \\
s=k_{1} y_{1}+b h+D \varepsilon(t)+j D \varepsilon(t),
\end{array}\right.
$$

and

$$
L 2:\left\{\begin{aligned}
\dot{x}_{1} & =a_{1}\left(x_{2}-x_{1}\right)+\left(s-s^{\prime}\right), \\
\dot{x}_{2} & =a_{2} x_{1}-x_{1} x_{3}-x_{2}+v_{2}, \\
\dot{x}_{3} & =-a_{3} x_{3}+(1 / 2)\left(\bar{x}_{1} x_{2}+x_{1} \bar{x}_{2}\right)+v_{3}, \\
s^{\prime} & =k_{1} x_{1}
\end{aligned}\right.
$$

where $h$ is the information signal, $b$ is its parameter, and $D \varepsilon(t)$ presents the white noise both arisen in the communication channel and produced by the noise source. The transmitted signal is $s$ and $s^{\prime}$ is the output of the receiver.

We get the error dynamics of real version from (11) and (12),

$$
\left\{\begin{array}{l}
\dot{e}_{u 1}=a_{1}\left(e_{u 3}-e_{u 1}\right)+b h^{r}+D \varepsilon(t)-\left(s^{r}-s^{\prime r}\right)=a_{1}\left(e_{u 3}-e_{u 1}\right)-k_{1} e_{u 1}, \\
\dot{e}_{u 2}=a_{1}\left(e_{u 4}-e_{u 2}\right)+b h^{i}+D \varepsilon(t)-\left(s^{i}-s^{\prime i}\right)=a_{1}\left(e_{u 3}-e_{u 1}\right)-k_{1} e_{u 2},
\end{array}\right.
$$


where the superscript $r$ stands for the real parts of the complex variables, while $i$ means the imaginary parts. Therefore, according to Theorem $2, \mathbf{x}=\left(x_{1}, x_{2}, x_{3}\right)^{\mathrm{T}}$ will approach $\mathbf{y}_{\tau}=\left(y_{1 \tau}, y_{2 \tau}, y_{3 \tau}\right)^{\mathrm{T}}$ as $t \rightarrow \infty$ and LS occurs.

Figure 1 shows the block diagram of our communication scheme. The transmitted signal is denoted as $s(t)=k_{1} y_{1}+b h+D \varepsilon(t)+j D \varepsilon(t)$. At the receiver end, the transmitted signal is denoted as $s(t-\tau)=k_{1} y_{1 \tau}+b h_{\tau}+D \varepsilon(t)+j D \varepsilon(t)$ because of the time lag of transmission (As the characteristics of white noise, we ignore the time lag of noise). According to Theorem 2, the controller is designed as

$$
v_{1}=s(t-\tau)-s^{\prime}, \quad v_{2}=k_{2}\left(y_{2 \tau}-x_{2}\right), v_{3}=k_{3}\left(y_{3 \tau}-x_{3}\right) .
$$

With the occurrence of LS, $\mathbf{x}$ will approach $\mathbf{y}_{\tau}$, and the mixed signal which includes noise $h_{g}=s(t-$ $\tau)-s^{\prime}=k_{1} e_{1}+D \varepsilon(t)+j D \varepsilon(t)+b h_{\tau} \rightarrow D \varepsilon(t)+j D \varepsilon(t)+b h_{\tau}$. As the recovered signal $h_{\tau}$ and the noise $D \varepsilon(t)$ are independent, we employ the method of independent component analysis (ICA) [28] to separate the noise from the recovered signal and extract the recovered information signal.

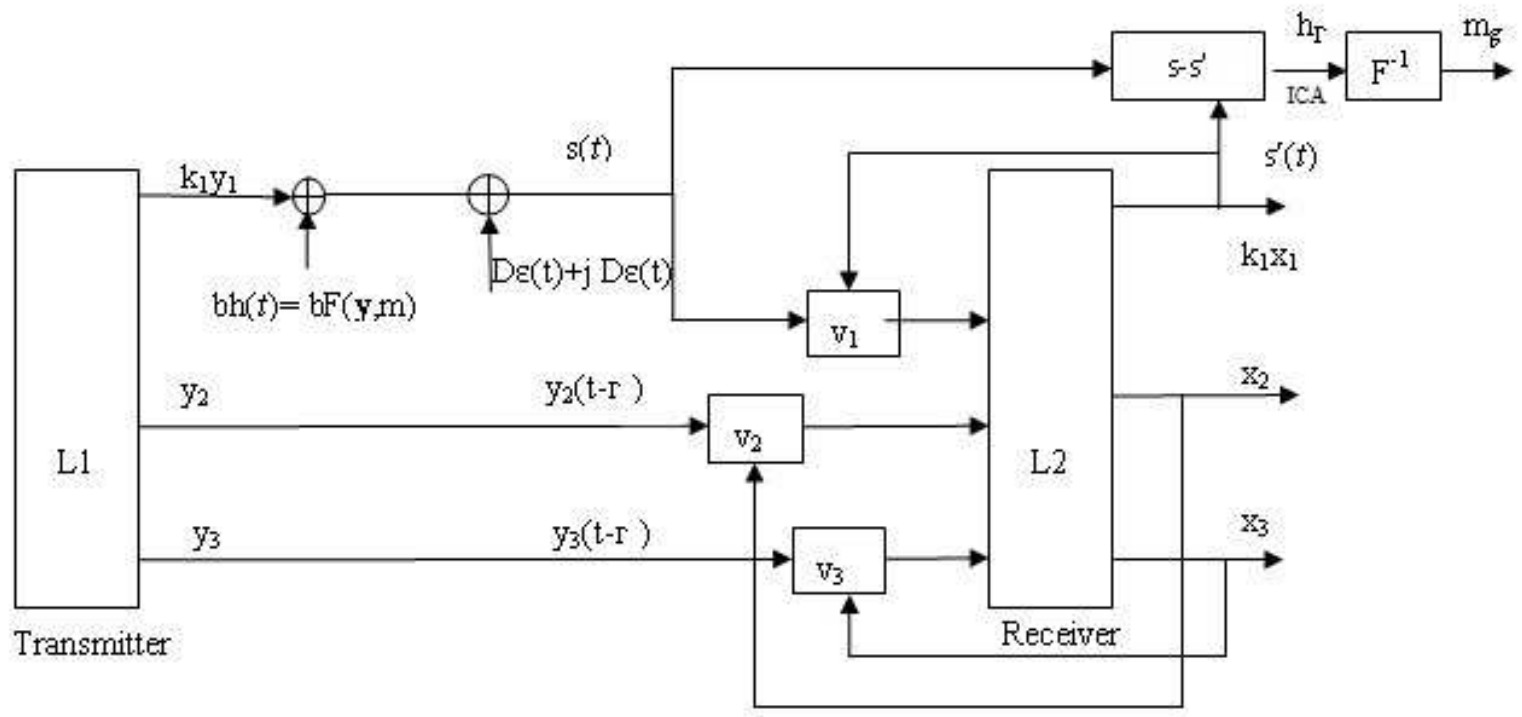

Figure 1. The block diagram of our communication scheme.

The mixed signal can be expressed by

$$
\left\{\begin{array}{l}
h_{g 1}=D \varepsilon(t)+j D \varepsilon(t)+b h_{\tau}, \\
h_{g 2}=D \varepsilon(t)+j D \varepsilon(t)+b^{\prime} h_{\tau},
\end{array}\right.
$$

where the parameter $b^{\prime} \neq b$. Then we can retrieve the recovered signal $h_{\tau}=\left(b-b^{\prime}\right)^{-1}\left(h_{g 1}-h_{g 2}\right)$. Obviously, we eliminate the effect of noise by ICA method.

In our communication scheme, we add the parameter $b$ or $b^{\prime}$ to regulate the amplitude of information signals, therefore, the amplitude of information signals can bigger than that of chaotic signals as long as the product of the amplitude of information signals and the parameter $b$ or $b^{\prime}$ is smaller than the amplitude of chaotic signals. It is easy to realize the parameter $b$ or $b^{\prime}$ by proportional regulator in practice. We also can employ $m(t)$ as the information signal in order to increase the security of this communication scheme, and $h(t)=F(\mathbf{y}, m)$ is a complicated function of information signal. 


\section{Simulations, Results and Discussion}

We adopt the system (11) as the transmitter and (12) as the receiver, and make simulations by using Matlab. We utilize the fourth-order Runge-Kutta scheme and $\Delta t$ is the reciprocal of the sampling frequency. We choose the initial condition $\mathbf{y}(0)=(2+1 j, 5+3 j, 4)^{\mathrm{T}}, \mathbf{x}(0)=(-1-2 j,-3-4 j, 1)^{\mathrm{T}}$ and the parameters $a_{1}=14, a_{2}=35, a_{3}=3.7$. The time-delay is $\tau=0.03 \mathrm{~s}$. The controller is (14).

The white noise $\varepsilon(t)$ is the stochastic Gaussian process. It can be depicted by the following probability distribution function

$$
q(\varepsilon)=\frac{1}{\sqrt{2 \pi} \sigma} \exp \left(-\frac{\left(\varepsilon-\varepsilon_{0}\right)^{2}}{2 \sigma^{2}}\right)
$$

where $\varepsilon_{0}=0$ and $\sigma=1$ are the mean value and variance, respectively [24].

We obtain the evolution of $\mathbf{x}(t)$ and $\mathbf{y}(t)$ from $5 \mathrm{~s}$ to $6 \mathrm{~s}$ shown as in Figure 2 with the control strength $k_{1}=20, k_{2}=k_{3}=10, D=5, b=200$ and $b^{\prime}=199$. The error vector of LS is shown in Figure 3 where the error vector converges to zero rapidly with time. It indicates that LS occurs. The controller $\mathbf{v}$ is displayed in Figure 4.

We transmit the famous melody "Ode to joy" which is written by L. Beethoven. It includes two components which can be read by Matlab software. They are shown in Figure 5a,b with the sampling frequency 44,100 Hz. They are carried by the real part and the imaginary part of $s(t)$, respectively. The SNR (signal-to-noise ratio $10 \mathrm{lg} S / N$ ) is $13 \mathrm{~dB}$ (where the amplitude of real signal is $b h(t)$ and the amplitude of noise is $D$ ). The SNR increases with $b$. For the signal with large amplitude, we can greatly decrease $b$ and retrieve the original signal accurately with low SNR under the effect of the ICA filter.
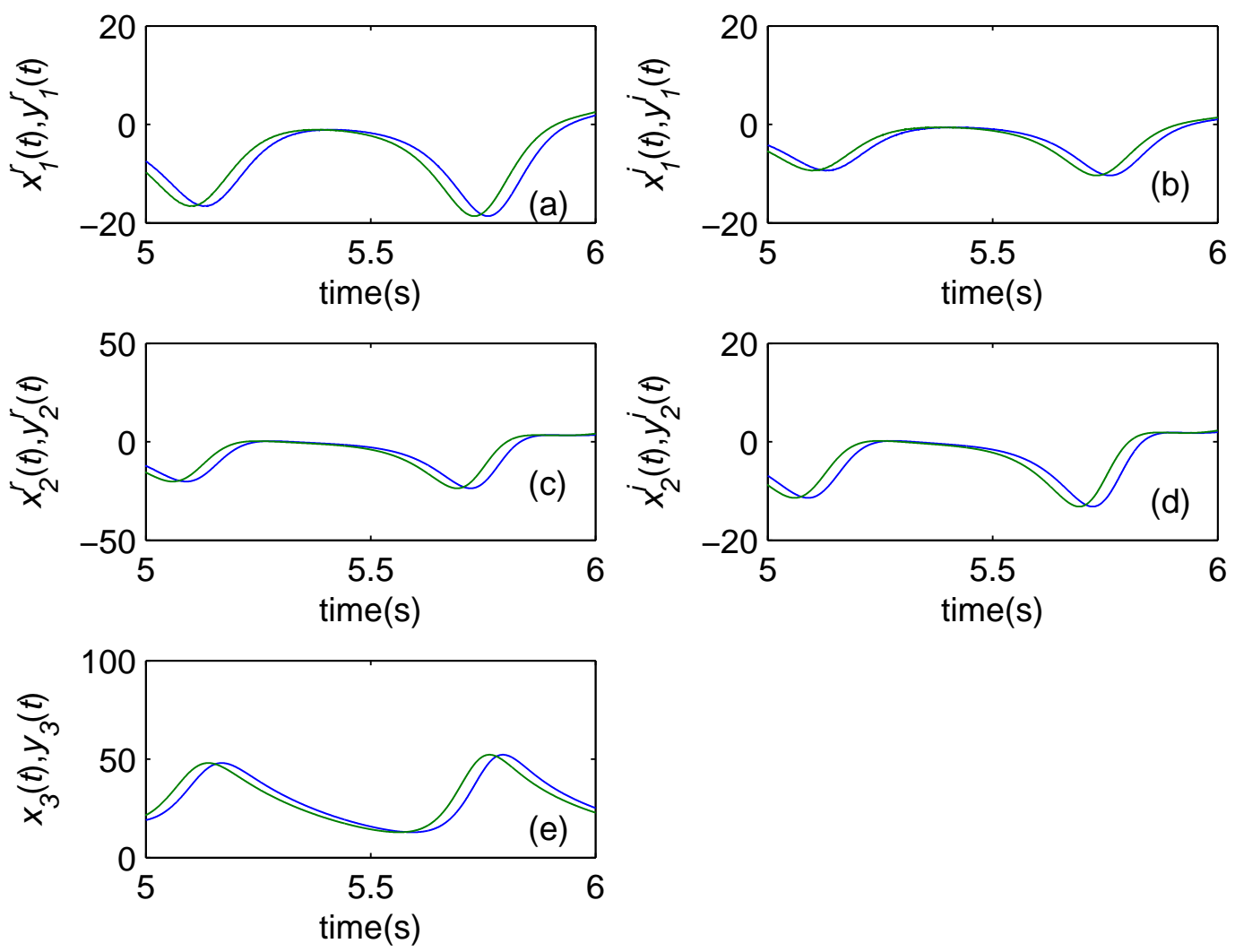

Figure 2. The evolution of $\mathbf{x}(t)$ and $\mathbf{y}(t)$ from $5 \mathrm{~s}$ to $6 \mathrm{~s}$. 

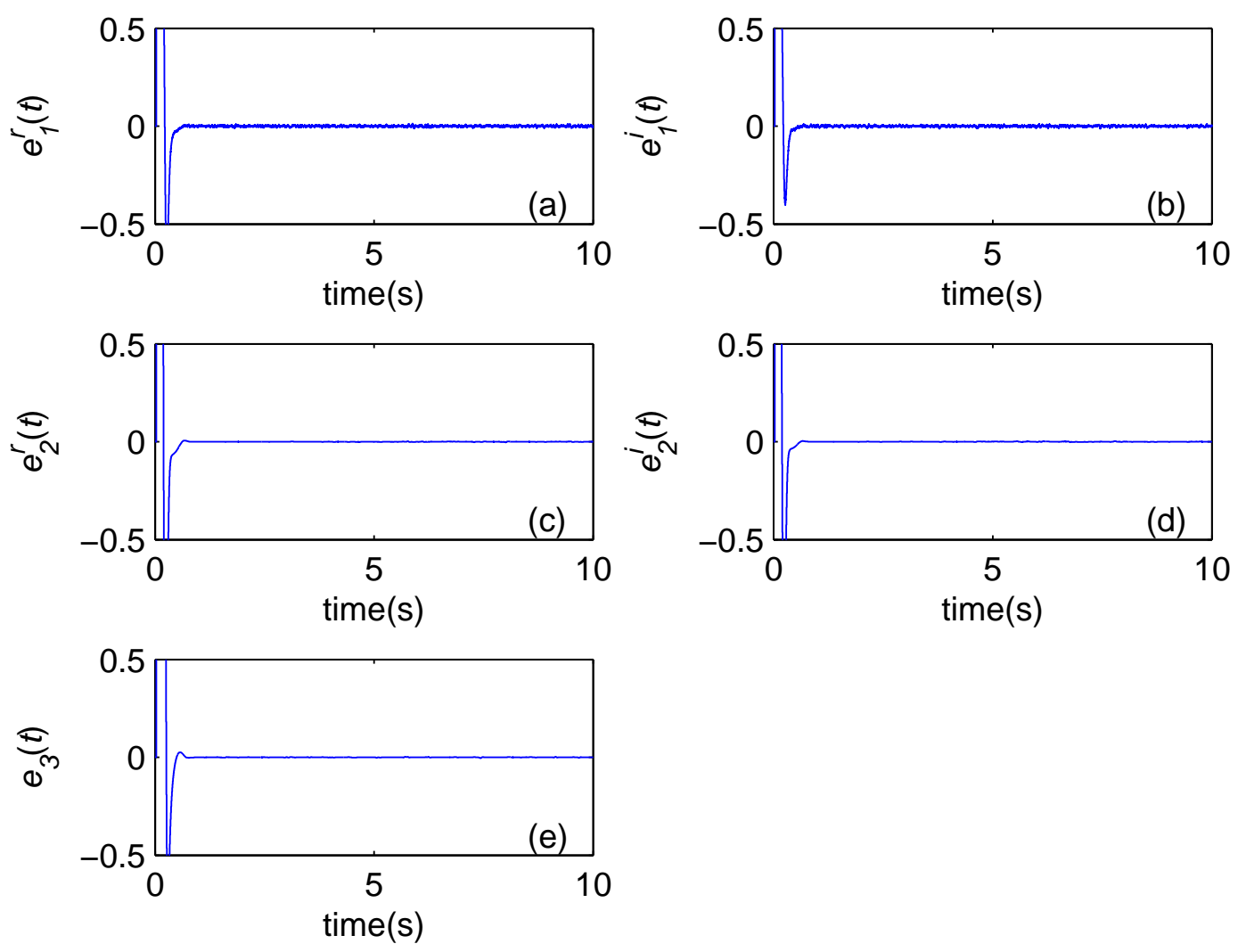

Figure 3. The error vector of LS.
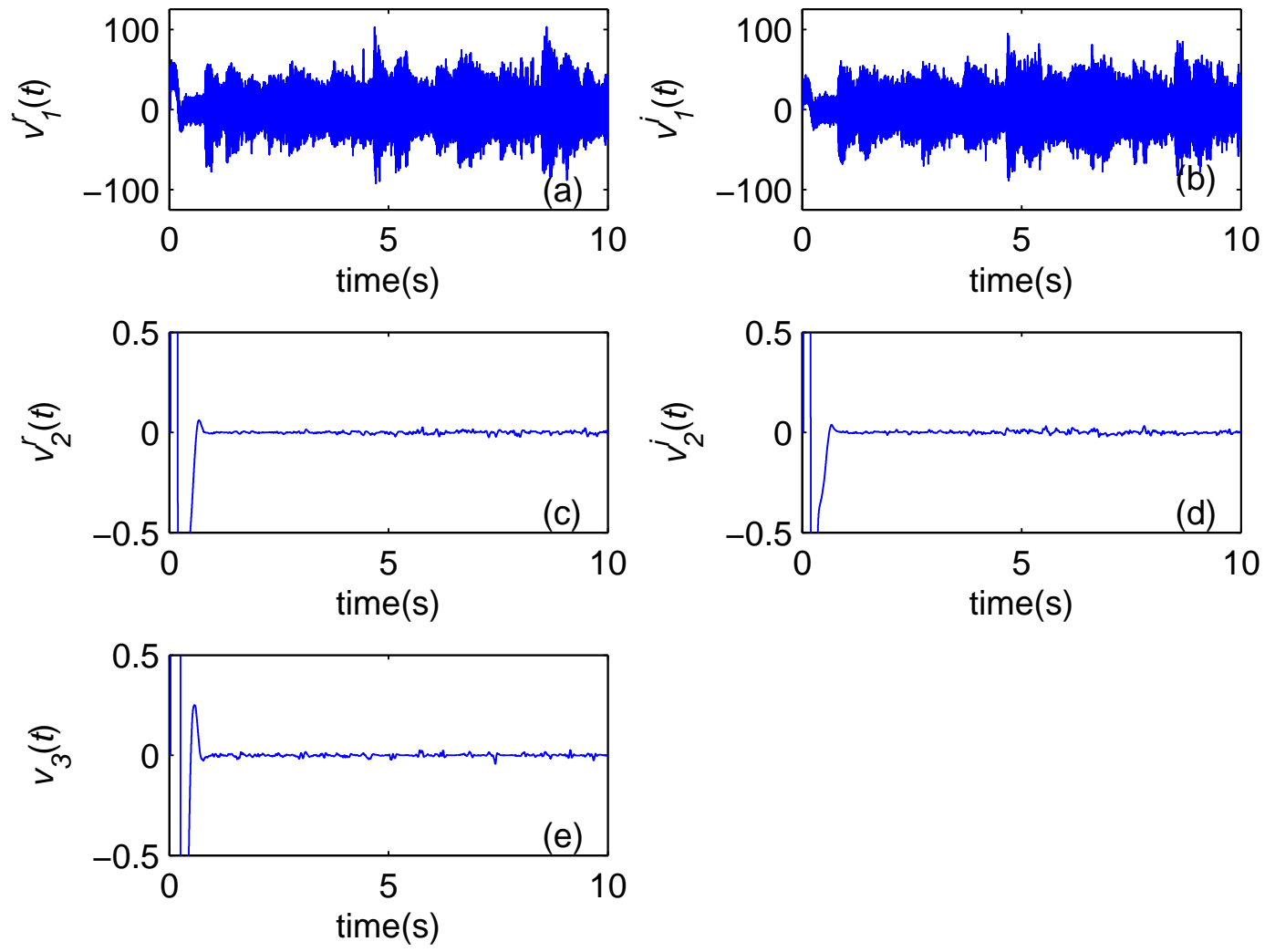

Figure 4. The LS controller. 

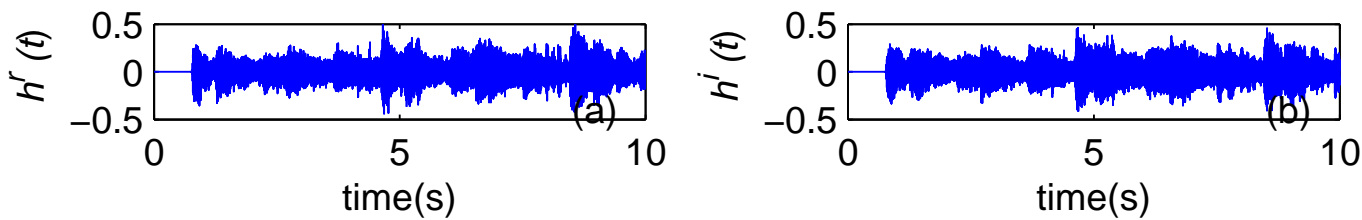

(c)
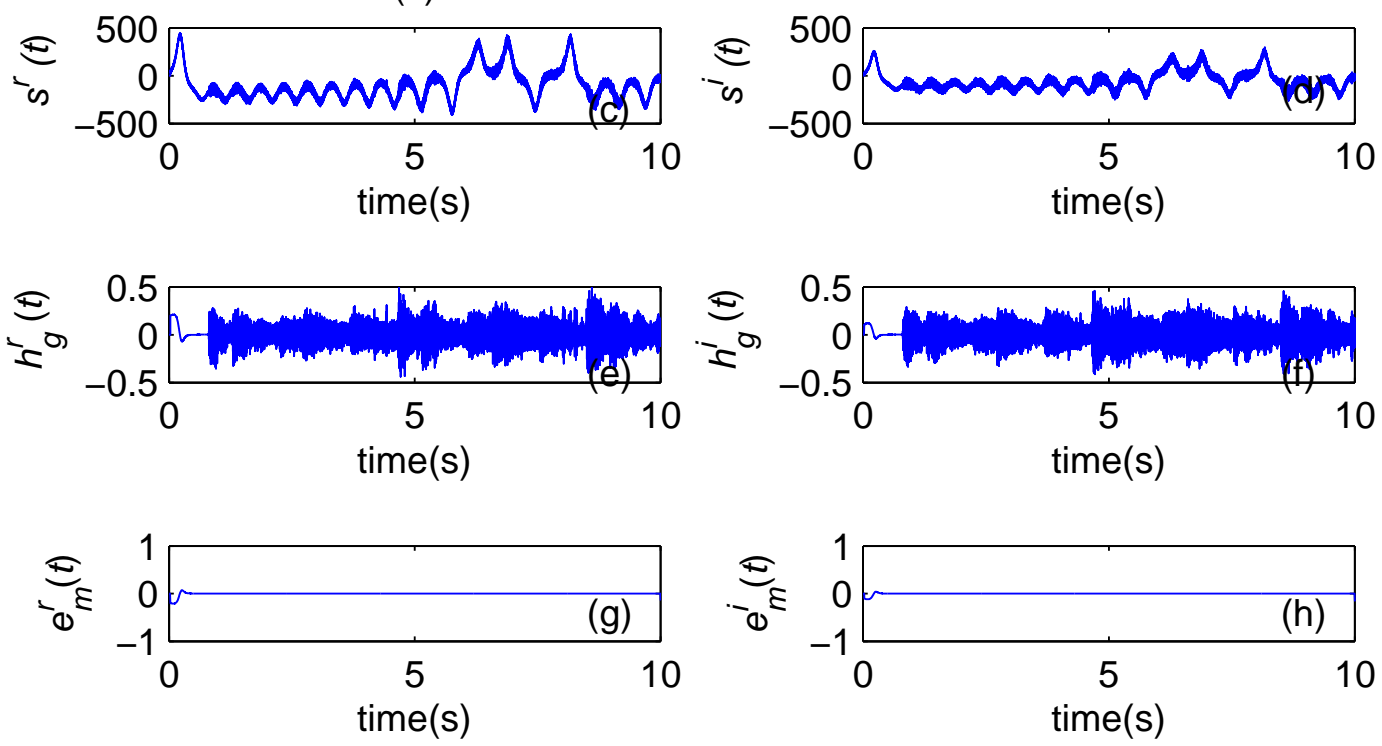

Figure 5. The transmission process of the famous melody "Ode to joy".

We obtain that the transmitted signal $s(t)$ completely conceals the information signal, which is depicted in Figure 5c,d. Obviously, $s(t)$ is a combination of state variables of drive system, information signal and white noise. For the signal with larger amplitude, we can decrease the value of $b$ to ensure that the chaotic signal masks the information signal. The recovered signal $h_{\tau}(t)$ are depicted in Figure 5e,f. It is easy to find that the information signal $h(t)$ is recovered accurately from Figure $5 \mathrm{~g}, \mathrm{~h}$.

Compared with other examples of communication system based on real chaotic system [20-30] and complex chaotic system [31-34], the above results of experimental simulations show the proposed secure communication scheme based on LS of complex chaotic system has many advantages.

(1) As the time delay of transmission, it is much closer to the real situation and more accurate to describe the synchronization between the transmitter and the receiver based on LS. It is more accurate and much closer to the real situation. This is one natural advantage in theory.

(2) It has double capacity of transmitted signals.

(3) The LS controller used in this communication scheme based on error feedback is easier than other techniques such as $[3,14]$ and it is easy to implement in practice, thus it has low amount of calculation, simplicity and practicability.

(4) Using the method of ICA, this communication scheme has a strong ability of anti-noise and robustness.

(5) Finally, we add the parameter $b$ to regulate the amplitude of information signals, thus this scheme can transmit multiple messages with all kinds of amplitudes. 
In a word, this communication scheme has simplicity, fast convergence speed, low amount of calculation, high capacity of transmitted signals, the ability of anti-noise, practicality and low SNR.

\section{Conclusion}

We design a LS controller of complex chaotic systems considering the time delay appears in the transmission in chaos-communication, then propose a communication scheme based on LS and ICA. The corresponding numerical simulations, where the complex Lorenz system are taken as a typical example, are implemented to demonstrate the effectiveness and feasibility of the proposed scheme.

\section{Acknowledgments}

The research was supported by the Nature Science Foundation of Shandong Province (numbers ZR2014FL015).

\section{Conflicts of Interest}

The author declares no conflict of interest.

\section{References}

1. Shahverdiev, E.M.; Sivaprakasam, S.; Shore, K.A. Lag synchronization in time-delayed systems. Phys. Lett. A 2002, 292, 320-324.

2. Taherion, S.; Lai, Y.C. Observability of lag synchronization of coupled chaotic oscillators. Phys. Rev. E 1999, 59, 6247-6250.

3. Mahmoud, E.E. Lag synchronization of hyperchaotic complex nonlinear systems via passive control. Appl. Math. Inf. Sci. 2013, 7, 1429-1436.

4. Zhang, Q.J.; Zhao, J.C. Projective and lag synchronization between general complex networks via impulsive control. Nonlinear Dyn. 2012, 67, 2519-2525.

5. Fowler, A.C.; Gibbon, J.D. The complex Lorenz equations. Physica D 1982, 4, 139-163.

6. Ning, C.Z.; Haken, H. Detuned lasers and the complex Lorenz equations: Subcritical and supercritical Hopf bifurcations. Phys. Rev. A 1990, 41, 3826-3837.

7. Mahmoud, G.M.; Bountis, T.; Mahmoud, E.E. Active control and global synchronization for complex Chen and Lü systems. Int. J. Bifurc. Chaos 2007, 17, 4295-4308.

8. Luo, C; Wang, X.Y. Chaos generated from the fractional-order complex Chen system and its application to digital secure communication. Int. J. Mod. Phys. C , 2013, 24, 1350025.

9. Mahmoud, G.M.; Mahmoud, E.E. Phase and antiphase synchronization of two identical hyperchaotic complex nonlinear systems. Nonlinear Dyn. 2010, 61, 141-152.

10. Mahmoud, G.M.; Mahmoud, E.E. Complete synchronization of chaotic complex nonlinear systems with uncertain parameters. Nonlinear Dyn. 2010, 62, 875-882.

11. Mahmoud, G.M.; Mahmoud, E.E. Synchronization and control of hyperchaotic complex Lorenz system. Math. Comput. Simul. 2010, 80, 2286-2296. 
12. Liu, S.T.; Liu, P. Adaptive anti-synchronization of chaotic complex nonlinear systems with unknown parameters. Nonlinear Anal. Real World Appl. 2011, 12, 3046-3055.

13. Liu, P.; Liu, S.T. Anti-synchronization between different chaotic complex systems. Phys. Scr. 2011, 83, 065006.

14. Mahmoud, G.M.; Mahmoud, E.E. Lag synchronization of hyperchaotic complex nonlinear systems. Nonlinear Dyn. 2012, 67, 1613-1622.

15. Liu, P.; Liu, S.T. Adaptive modified function projective synchronization of general uncertain chaotic complex systems. Phys. Scr. 2012, 85,035005.

16. Liu, P.; Liu, S.T. Robust adaptive full state hybrid synchronization of chaotic complex systems with unknown parameters and external disturbances. Nonlinear Dyn. 2012, 70, 585-599.

17. Mahmoud, E.E. Modified projective phase synchronization of chaotic complex nonlinear systems. Math. Comput. Simul. 2013, 89, 69-85.

18. Zhang, F.F.; Liu, S.T.; Yu, W.Y. Modified projective synchronization with complex scaling factors of uncertain real chaos and complex chaos. Chin. Phys. B 2013, 22, 120505.

19. Zhang, F.F.; Liu, S.T. Full State Hybrid Projective Synchronization and Parameter Identification for Uncertain Chaotic (Hyperchaotic) Complex Systems. J. Comput. Nonlin. Dyn. 2013, 9, 021009.

20. Li, Z.G.; Xu, D.L. A secure communication scheme using projective chaos synchronization. Chaos Soliton Fractals 2004, 22,477-481.

21. Chee, C.Y.; Xu, D.L. Secure digital communication using controlled projective synchronisation of chaos. Chaos Soliton Fractals 2005, 23, 1063-1070.

22. Hoang, T.M.; Nakagawa, M. A secure communication system using projective-lag and/or projective-anticipating synchronizations of coupled multidelay feedback systems. Chaos Soliton Fractals 2008, 38, 1423-1438.

23. Zheng, G.; Boutat, D.; Floquet, T.; Barbot, J.P. Secure communication based on multi-input multi-output chaotic system with large message amplitude. Chaos Soliton Fractals 2008, 41 1510-1517.

24. Moskalenko, O.I.; Koronovskii, A.A.; Hramov, A.E. Generalized synchronization of chaos for secure communication: remarkable stability to noise. Phys. Lett. A 2010, 374, 2925-2931.

25. Wang, X.Y.; Gao, Y.F. A switch-modulated method for chaos digital secure communication based on user-defined protocol. Commun. Nonlinear Sci. Numer. Simul. 2010, 15, 99-104.

26. Wang, X.Y.; Xu, B.; Zhang, H.G. A multi-ary number communication system based on hyperchaotic system of 6th-order cellular neural network. Commun. Nonlinear Sci. Numer. Simul. 2010, 15, 124-133.

27. Liu, H.J.; Wang, X.Y.; Zhu, Q.L. Asynchronous anti-noise hyper chaotic secure communication system based on dynamic delay and state variables switching. Phys. Lett. A 2011, 375, 2828-2835.

28. Zhang, Y.Q.; Wang, X.Y. A parameter modulation chaotic secure communication scheme with channel noises. Chin. Phys. Lett., 2011, 28, 020505.

29. Eisencraft, M.; Fanganiello, R.D.; Grzybowski, J.M.V.; Soriano, D.C.; Attux, R.; Batista, A.M.; Macau, E.E.N.; Monteiro, L.H.A.; Romano, J.M.T.; Suyama, R.; et al. Chaos-based communication systems in non-ideal channels. Commun. Nonlinear Sci. Numer. Simul. 2012, 17, 4707-4718. 
30. Wang, X.Y.; Xu, Bing; Luo, C. An asynchronous communication system based on the hyperchaotic system of 6th-order cellular neural network. Opt. Commun. 2012, 285, 5401-5405

31. Wang, X.Y.; Luo, C. Hybrid Modulus-phase Synchronization of Hyperchaotic Complex Systems and its Application to Secure Communication. Int. J. Nonlinear Sci. Numer. Simul. 2013, 14, 533-542

32. Mahmoud, G.M.; Mahmoud, E.E.; Arafa, A.A. On projective synchronization of hyperchaotic complex nonlinear systems based on passive theory for secure communications. Phys. Scr. 2013, 87, 055002.

33. Liu, S.T.; Zhang, F.F. Complex function projective synchronization of complex chaotic system and its applications in secure communication. Nonlinear Dyn. 2014, 76, 1087-1097.

34. Zhang, F.F.; Liu, S.T. Self-time-delay synchronization of time-delay coupled complex chaotic system and its applications to communication. Int. J. Mod. Phys. C 2014, 25, 1350102.

35. Liu, P.; Liu, S.T. Control and coupling synchronization of Julia sets in coupled map lattice. Indian J. Phys. 2012, 86, 455-462.

36. Zhang, Y.P.; Qiao, W.; Sun, J. Control and synchronization of Julia sets of complex standard family. Indian J. Phys. 2013, 87, 271-274.

37. Gibbon, J.D.; McGuinnes, M. The real and complex Lorenz equations in rotating fluids and laser. Physica D 1982, 5, 108-122.

38. Rauh, A.; Hannibal, L.; Abraham, N.B. Global stability properties of the complex Lorenz model. Physica D 1996, 99, 45-58.

39. Richter, H. Controlling the Lorenz system: Combining global and local schemes. Chaos Soliton Fractals 2001, 12, 2375-2380.

40. Mahmoud, G.M.; Aly, S.A. Farghaly, A.A. On chaos synchronization of a complex two coupled dynamos system. Chaos Soliton Fractals 2007, 33, 178-187.

41. Mahmoud, G.M.; Al-Kashif, M.A.; Farghaly, A.A. Chaotic and hyperchaotic attractors of a complex nonlinear system. J. Phys. A 2008, 41, 055104.

42. Mahmoud, G.M.; Ahmed, M.E.; Mahmoud, E.E. Analysis of hyperchaotic complex Lorenz systems. Int. J. Mod. Phys. C 2008, 19, 1477-1494.

43. Mahmoud, G.M.; Bountis, T.; AbdEl-Latif, G.M.; Mahmoud, E.E. Chaos synchronization of two different complex Chen and Lü systems. Nonlinear Dyn. 2009, 55, 43-53.

44. Fang, N.W. Eigenvalues of a class of diagonally dominant matrics and application. J. Anhui Univ. 1995, 1, 18-22. (In Chinese)

(c) 2015 by the author; licensee MDPI, Basel, Switzerland. This article is an open access article distributed under the terms and conditions of the Creative Commons Attribution license (http://creativecommons.org/licenses/by/4.0/). 